\title{
Quasi-localized wavefunctions on magnetized tori and tiny neutrino Yukawa couplings
}

\author{
Keigo Sumita \\ Department of Physics, Waseda University, \\ Tokyo 169-8555, Japan \\ E-mail: k.sumita@aoni.waseda.jp
}

ABstRACT: This paper shows that, a quasi-localization of wavefunctions in toroidal compactifications with magnetic fluxes can lead to a strong suppression for relevant Yukawa couplings, and it is applicable to obtain tiny neutrino masses. Although it is known that magnetic fluxes lead to a Gaussian profile of zero-modes on a torus and that can yield a suppressed coupling in higher-dimensional supersymmetric Yang-Mills (SYM) theories, the largest (diagonal) entry of Yukawa matrices is always of $\mathcal{O}(1)$. In this paper, we propose a way to induce an absolutely tiny global factor of Yukawa matrices. In two SYM theories defined in different dimensional spacetime, their bifundamental representations must be localized as a point in some directions. Overlaps of such point-like localized wavefunctions and Gaussian zero-modes give a global factor of Yukawa matrices, and it can be a strong suppression factor or a usual $\mathcal{O}(1)$ factor, corresponding to their distance. Our numerical analysis shows that it is possible to obtain a suppression strong enough to realize the tiny neutrino masses without a fine-tuning. Furthermore, we propose a concrete model in a magnetized SYM system and demonstrate the mechanism to generate the tiny neutrino Yukawa couplings.

KEYwords: Phenomenology of Field Theories in Higher Dimensions

ARXIV EPRINT: 1509.03392 


\section{Contents}

1 Introduction 1

2 Toroidal compactification with magnetic fluxes 2

3 Suppression factor in mixed SYM systems 5

$\begin{array}{llr}4 & \text { A concrete model } & 8\end{array}$

5 Conclusions and discussions 11

\section{Introduction}

Extra dimensional space is an attractive candidate for new physics beyond the standard model (SM). This has been being studied very actively since Kaluza-Klein theory was proposed to unify the forces of nature. Although our circumstances has got more complicated than those at that time, such a unified theory is expected to contain the extra dimensional space which is an origin of many flavors and the forces of the SM. Indeed, superstring theories which are candidates for the unified theory are defined in ten-dimensional spacetime, and the existence of extra dimensional space is indispensable for realizing the flavor structure in those theories. Higher-dimensional supersymmetric Yang-Mills (SYM) theories are expected as well-motivated higher-dimensional field theories since they appear in lowenergy limits of superstring theories. They are also attractive from the phenomenological viewpoint even if we do not consider superstring theories. The reason is that they are the most simple theories containing supersymmetry (SUSY) which is another great candidate for the new physics as well as the extra dimensional space, and the SM can be embedded into the SYM theories with a large gauge group.

Toroidal compactifications with magnetic fluxes are able to yield the three forces and the flavor structure of the SM in superstring theories and higher-dimensional SYM theories $[1,2]$. Magnetic fluxes on two-dimensional (2D) tori lead to generations of the quarks and the leptons, and the Higgs fields as degenerate zero-modes. Zero-mode equations for these fields can be solved analytically thanks to simplicity of tori, and zero-mode wavefunctions are obtained as solutions of the equations. We see from the wavefunctions that the zero-modes are quasi-localized on the magnetized torus with a Gaussian profile. When zero-modes are localized far away from each other, their four-dimensional (4D) effective coupling is suppressed [3]. Thus, magnetic fluxes on tori can induce hierarchical Yukawa couplings, and the hierarchical masses and mixing angles of the quarks and the leptons could be potentially obtained on the magnetized torus. Indeed, a semi-realistic pattern of them was realized in ref. $[4,5]$. 
The tiny neutrino mass is a remaining mystery of the SM. Although the magnetized toroidal compactifications can yield suppressed Yukawa couplings, the largest diagonal entry of Yukawa matrices is always estimated to be of $\mathcal{O}(1)$, and another mechanism to realize the tiny masses is needed. An elegant solution for explaining the tiny masses is given by introducing heavy Majorana mass terms for the right-handed neutrinos, which is a so-called seesaw mechanism [6-8]. However, there is a problem in toroidal magnetized compactification of SYM theories. Such a Majorana mass term cannot be obtained straightforwardly in the theories. We are forced to assume that such mass terms might be generated by nonperturbative effects $[9,10]$ and/or higher-dimensional operators. Although there is a wide variety of the seesaw mechanisms, they all require the presence of additional mass terms. Thus, this paper aim to propose a simple way to realize tiny Yukawa couplings for the neutrinos in the magnetized toroidal compactifications.

This paper is organized as follow. Section 2 is devoted to review the magnetized toroidal compactifications in SYM theories. Zero-mode wavefunctions on the magnetized torus and their leading Yukawa couplings are shown. We give the main idea and result of this paper in section 3. A numerical analysis is also performed to verify consistency with the tiny neutrino masses. In section 4 , we propose a concrete model with a specific configuration of magnetic fluxes on the tori in an SYM system, where our mechanism to yield the tiny neutrino Yukawa couplings will work correctly. We conclude with a discussion of prospects in section 5 .

\section{Toroidal compactification with magnetic fluxes}

This section gives an overview of magnetized toroidal compactifications on the basis of sixdimensional (6D) SYM theories compactified on a two-dimensional (2D) torus, following ref. [9] (See also [11] for a review of magnetized toroidal compactifications). In this section, we use complex coordinates $z$ and a complex 2D vector field $A_{z}$ defined as

$$
\begin{array}{rlrl}
z & \equiv \frac{1}{2}\left(x^{1}+\tau x^{2}\right), & \bar{z} \equiv(z)^{*}, \\
A_{z} \equiv-\frac{1}{\operatorname{Im} \tau}\left(\tau^{*} A_{4}-A_{5}\right), & \bar{A}_{\bar{z}} \equiv\left(A_{z}\right)^{\dagger},
\end{array}
$$

where $\left(x^{1}, x^{2}\right)$ are the real torus coordinates and $\left(A_{4}, A_{5}\right)$ are extra dimensional components of a (real) $6 \mathrm{D}$ vector field. Periodicity of this torus is expressed by identifications of $z \sim z+1$ and $z \sim z+\tau$. Complex structure of this torus is denoted by $\tau$. This is defined with the torus radius $R$ in the torus metric as

$$
d s^{2}=g_{a b} d x^{a} d x^{b} \equiv 2 h d z d \bar{z},
$$

where

$$
g=(2 \pi R)^{2}\left(\begin{array}{cc}
1 & \operatorname{Re} \tau \\
\operatorname{Re} \tau & |\tau|^{2}
\end{array}\right), \quad h=2(2 \pi R)^{2} .
$$

On this torus, we consider the following gauge potential containing magnetic fluxes and Wilson lines,

$$
A_{z}=\frac{\pi}{\operatorname{Im} \tau}(M \bar{z}+\zeta)
$$


The magnetic fluxes and Wilson lines are represented by $(N \times N)$-matrices in $\mathrm{U}(N) \mathrm{SYM}$ theories. Since this paper concentrates on Abelian forms of magnetic fluxes and Wilson lines, the matrices take nonvanishing values only for their diagonal entries. Note that the nonvanishing entries of magnetic fluxes are forced to be integer because of the Dirac's quantization condition.

We discuss gauge symmetry breaking due to these magnetic fluxes and Wilson lines. In the case of $\mathrm{U}(2)$ SYM theory, this $\mathrm{U}(2)$ gauge symmetry is broken down to $\mathrm{U}(1) \times \mathrm{U}(1)$ when the two diagonal elements of magnetic fluxes (Wilson lines) are chosen to be different values. This is extended to the case of $\mathrm{U}(N)$ SYM theories, and the gauge symmetry is broken as $\mathrm{U}(N) \rightarrow \prod_{a} \mathrm{U}\left(N_{a}\right)$, where $\mathrm{U}\left(N_{a}\right)$ is an unbroken gauge subgroup of $\mathrm{U}(N)$.

We consider zero-mode equations for a two-component spinor field on this magnetized torus. Fields living in extra dimensional space are decomposed into the zero-mode and multiple Kaluza-Klein modes, and we focus on the zero-mode in the following. The twocomponent spinor is denoted by

$$
\psi=\left(\begin{array}{l}
\psi_{+} \\
\psi_{-}
\end{array}\right),
$$

and then, their zero-mode equations are given by

$$
\begin{aligned}
& \bar{\partial}_{z} \psi_{+}+\left[\bar{A}_{\bar{z}}, \psi_{+}\right]=0, \\
& \partial_{z} \psi_{-}-\left[A_{z}, \psi_{-}\right]=0 .
\end{aligned}
$$

In the assumption of gauge symmetry breaking $\mathrm{U}(N) \rightarrow \prod_{a} \mathrm{U}\left(N_{a}\right)$, a bifundamental representation $\left(N_{a}, \bar{N}_{b}\right)$ of two remaining gauge subgroups $\mathrm{U}\left(N_{a}\right) \times \mathrm{U}\left(N_{b}\right)$ contained in $\psi_{ \pm}$ is denoted by $\psi_{ \pm}^{a b}$. Their zero-mode equations are given by

$$
\begin{aligned}
& {\left[\bar{\partial}_{z}+\frac{\pi}{2 \operatorname{Im} \tau}\left(M_{a b} z+\zeta_{a b}\right)\right] \psi_{+}^{a b}=0,} \\
& {\left[\partial_{z}-\frac{\pi}{2 \operatorname{Im} \tau}\left(M_{a b} \bar{z}+\bar{\zeta}_{a b}\right)\right] \psi_{-}^{a b}=0,}
\end{aligned}
$$

where $M_{a b} \equiv M_{a}-M_{b}$ and $\zeta_{a b} \equiv \zeta_{a}-\zeta_{b}$, and $M_{a}$ and $\zeta_{a}$ are relevant entries of $M$ and $\zeta$.

These equations were studied in detail in ref. [2]. Either the zero-mode equations for $\psi_{+}^{a b}$ or $\psi_{-}^{a b}$ gives a well-defined wavefunction, which is related to the sign of magnetic fluxes $M_{a b}$. In the case of $M_{a b}>0$, a bifundamental $\psi_{+}^{a b}$ has normalizable solutions in the zero-mode equation (2.1), and then, zero-modes of $\psi_{-}^{a b}$ are eliminated. There appear only the zero-modes of $\psi_{+}^{a b}$ with a degeneracy given by $M_{a b}$. In contrast, the zero-mode equation (2.2) yields the zero-modes of $\psi_{-}^{a b}$ when $M_{a b}<0$, and its degeneracy is given by $\left|M_{a b}\right|$. This is a kind of chirality projection to yield a $4 \mathrm{D}$ chiral spectrum like the SM. Furthermore, we can identify these degenerate zero-modes with the generations of the SM particles. $\left|M_{a b}\right|=3$ unit of magnetic flux leads to the three generations of quarks and leptons.

Their $4 \mathrm{D}$ effective coupling constants are of our main interest because they would be Yukawa couplings of the SM which determine the masses and mixing angles of the quarks and leptons. The coupling constant of $4 \mathrm{D}$ effective field theories is given as an overlap 
integral of wavefunctions on the torus. Thus, we are required to study the zero-mode wavefunctions obtained as solutions of eqs. (2.1) and (2.2). The zero-mode wavefunctions are obtained analytically, which are expressed by using Jacobi-theta functions. We use an index $I_{a b}$ to label the $\left|M_{a b}\right|$ degenerate zero-modes, that is, $I_{a b}=1,2, \ldots,\left|M_{a b}\right|$. The wavefunction of the $I_{a b}$-th zero-mode $\psi_{+}^{I_{a b}}$ for $M_{a b}>0$ is then given by

$$
\begin{aligned}
\psi_{+}^{I_{a b}} & =\Theta^{I_{a b}, M_{a b}}\left(z+\zeta_{a b} / M_{a b}, \tau\right), \\
\Theta^{I, M}(z, \tau) & =\mathcal{N}_{M} e^{\pi i M z \operatorname{Im} z / \operatorname{Im} \tau} \vartheta\left[\begin{array}{c}
I / M \\
0
\end{array}\right](M z, M \tau),
\end{aligned}
$$

where the Jacobi-theta function is defined by

$$
\vartheta\left[\begin{array}{l}
a \\
b
\end{array}\right](\nu, \tau)=\sum_{\ell \in \mathbb{Z}} e^{\pi i(a+\ell)^{2} \tau} e^{2 \pi i(a+\ell)(\nu+b)} .
$$

Normalization factors are determined as

$$
\int d z d \bar{z} \sqrt{\operatorname{det} g} \Theta^{I, M}\left(\Theta^{J, M}\right)^{*}=\delta_{I J}
$$

and we find

$$
\mathcal{N}_{M}=\left(\frac{2 \operatorname{Im} \tau|M|}{\mathcal{A}^{2}}\right)^{1 / 4}
$$

where $\mathcal{A}$ denotes the area of torus. For $M_{a b}<0$, the wavefunction of $\psi_{-}^{I_{a b}}$ is given by

$$
\psi_{-}^{I_{a b}}=\left(\Theta^{I_{a b}, M_{a b}}\left(z+\zeta_{a b} / M_{a b}, \tau\right)\right)^{*} .
$$

These zero-modes have a Gaussian profile in the extra dimensional space. For instance, we depict the squared absolute values of three wavefunctions $\left|\psi_{+}^{I_{a b}}\right|^{2}$ given by a parameter set of $\left(M_{a b}=3, \zeta_{a b}=0, \tau=i\right)$ in a direction of the torus $(\operatorname{Re} z=0)$ in figure 1 . Three colored lines correspond to the three zero-modes labeled by $I_{a b}=1,2,3$. We see from this figure that the degenerate zero-modes are quasi-localized at points of the torus, and their peak positions are different from each other. These peak positions can be shifted by introducing non-vanishing Wilson lines $\zeta_{a b} \neq 0$. As we said above, Yukawa couplings of the SM are expected to be given by an overlap integral of the zero-mode wavefunctions of a left-handed matter field, a right-handed matter field and a Higgs field. When their wavefunctions are localized far away from each other on the magnetized torus, a suppressed Yukawa coupling will be obtained. This is able to lead to hierarchical Yukawa couplings. Indeed, a semi-realistic pattern of the masses and mixing angles of the quarks and the leptons was realized without hierarchical input parameters $[4,5]$.

Yukawa couplings can be calculated analytically by performing overlap integrals. They originate from the higher-dimensional gauge coupling, and the overlap integral is typically given by

$$
\begin{aligned}
Y_{I_{a b} J_{b c} K_{c a}}=g \int_{T^{2}} d z d \bar{z} \Theta^{I_{a b}, M_{a b}}\left(z+\zeta_{a b} / M_{a b}, \tau\right) & \\
& \times \Theta^{J_{b c}, M_{b c}}\left(z+\zeta_{b c} / M_{b c}, \tau\right) \Theta^{K_{c a}, M_{c a}}\left(z+\zeta_{c a} / M_{c a}, \tau\right),
\end{aligned}
$$




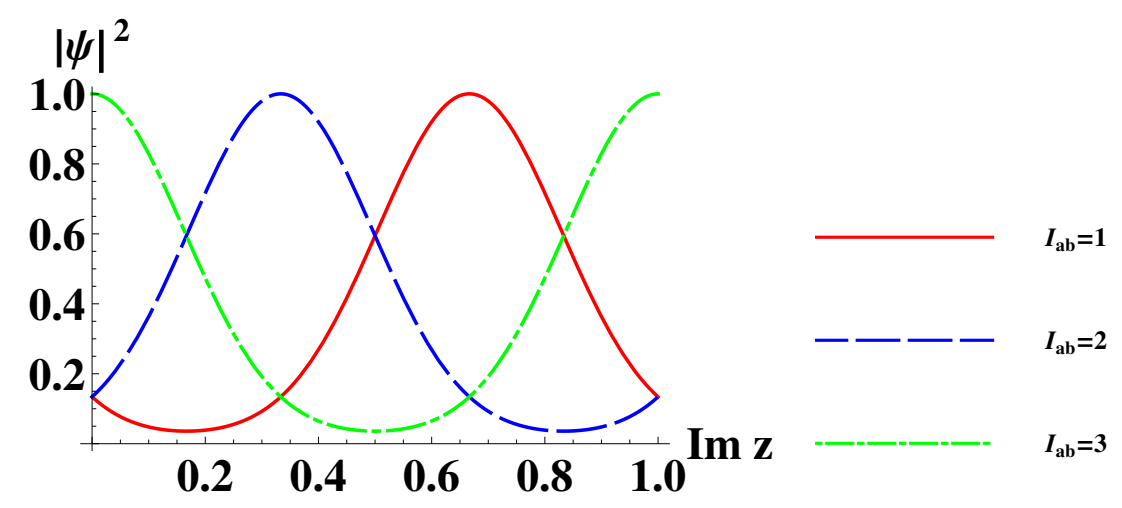

Figure 1. The squared absolute values of wavefunctions of the three zero-modes $\left|\psi_{+}^{I_{a b}}\right|^{2}$ given in eq. (2.3) with $\left(M_{a b}=3, \zeta_{a b}=0, \tau=i\right)$ are shown, where we set $\operatorname{Re} z=0$ and the horizontal axis corresponds to the other direction of torus.

where $g$ is the higher-dimensional gauge coupling constant. Ref. [2] performed this integral and found the result as

$$
\begin{aligned}
Y_{I_{a b} J_{b c} K_{c a}=} & g \frac{\mathcal{N}_{M_{a b}} \mathcal{N}_{M_{b c}}}{\mathcal{N}_{M_{c a}}} \exp \left\{\frac{\pi i}{\operatorname{Im} \tau}\left[\zeta_{a b} \operatorname{Im} \frac{\zeta_{a b}}{M_{a b}}+\zeta_{b c} \operatorname{Im} \frac{\zeta_{b c}}{M_{b c}}+\zeta_{c a} \operatorname{Im} \frac{\zeta_{c a}}{M_{c a}}\right]\right\} \\
& \times \sum_{m \in \mathbb{Z}_{M_{a c}}} \vartheta\left[\begin{array}{c}
\frac{M_{b c} I_{a b}-M_{a b} J_{b c}+M_{a b} M_{b c} m}{M_{a b} M_{b c} M_{a c}} \\
0
\end{array}\right]\left(M_{b c} \zeta_{a b}-M_{a b} \zeta_{b c}, \tau M_{a b} M_{b c} M_{a c}\right) \\
& \times \delta_{I_{a b}+J_{b c}+M_{a b} m, K_{c a}},
\end{aligned}
$$

for $M_{a b}, M_{b c}>0$ and $M_{c a}<0$. In evaluating this result numerically, we see that the Yukawa couplings can be hierarchical enough to realize the ratio of masses of the top and up quarks $\left(\sim \mathcal{O}\left(10^{-5}\right)\right)$. However, the largest entry of $Y_{I_{a b} J_{b c} K_{c a}}$, which is identified with a $(3,3)$ entry of Yukawa matrices, is obtained to be of $\mathcal{O}(1)$ invariably. This is because as follows. In the magnetized models, overlaps of gaussian wavefunctions of the matters and Higgs fields determine the Yukawa couplings, and the gaussians of three generations are localized with a constant interval in the compact space shown as figure 1. As a result, one of the three generations must be localized near that of the Higgs fields, and the largest entry of Yukawa couplings is always almost unity. Thus, tiny neutrino masses $(\nu \lesssim \mathcal{O}(1 \mathrm{eV}))$ cannot be obtained with these Yukawa couplings, without heavy Majorana masses for the right-handed neutrinos [6-8]. (The masses of the bottom and tau can be realized by using the degree of freedom of $\tan \beta \equiv\left\langle H_{u}\right\rangle /\left\langle H_{d}\right\rangle$, where $H_{u}\left(H_{d}\right)$ represents the up- (down-) type Higgs field.) This paper provides a way to yield the absolutely tiny Yukawa couplings in the magnetized toroidal compactifications, which has a global strong suppression factor.

\section{Suppression factor in mixed SYM systems}

This section explains the basic idea for realizing a global factor to strongly suppress the Yukawa couplings. In the following, we will consider generic $(4+2 n)$-dimensional SYM 
theories compactified on magnetized tori, where the net number of degenerate zero-modes of a bifundamental representation is given by a product of relevant magnetic fluxes on each torus,

$$
N_{a b}=\prod_{i=1}^{n}\left|M_{a b}^{(i)}\right| .
$$

Three-generation structure of matter fields must originate from a single torus, and then, magnetic fluxes on the other tori are required not to generate extra zero-modes (i.e., $N_{a b}=$ $3 \times 1 \times 1)$. Furthermore, all the generation structure of the SM must originate from the same single torus, otherwise the rank of Yukawa matrices is to be reduced to one and some of matter fields would remain massless after the electroweak symmetry breaking.

In such three-generation models, the Yukawa couplings have additional global factors originating from the extra tori where the magnetic fluxes lead to just one generation. Although such a global factor can take a small value depending on the localization of the gaussian wavefunctions, it is difficult to construct a specific model for the following technical reason. There are a few allowed configurations of magnetic fluxes which will not induce extra generations of matter fields. They are represented by $\left(M_{Q_{L}}, M_{Q_{R}}, M_{H}\right)=( \pm 1, \mp 1,0)$ or $( \pm 1, \pm 1, \mp 2)$, where $M_{Q_{L}}, M_{Q_{R}}$ and $M_{H}$ are magnetic fluxes felt by left- and righthanded matter and Higgs fields. In the former configuration, one generation of matters and Higgs multiplets are generated, but one of the wavefunctions become a constant because of the vanishing flux. The global factor is then given by eq. (2.5) which cannot be small. In the latter case, the magnetic fluxes double the number of Higgs fields. As a result, unsuppressed Yukawa couplings will necessarily remain, which is the same discussion as that given below eq. (2.7).

In the following, we consider a mixture of two SYM theories defined in different dimensions of spacetime to get the strong suppression, especially, we focus on a system consisting of $6 \mathrm{D}$ and 10D SYM theories which is well motivated by stable D-brane systems in type IIB superstring theory. Such a mixed configurations generically contains bifundamental representations charged under both the 6D and 10D SYM theories. Zero-mode wavefunction of such bifundamentals depend on all the 10D coordinates, but they must be localized as a point in four extra directions where the 6D SYM fields cannot move.

We focus on a Yukawa coupling among the two bifundamental fields and an adjoint field of 10D SYM theory. The two bifundamental fields should be localized at a point in the four direction but the 10D adjoint field has a Gaussian profile on the magnetized tori. This Yukawa coupling in the $4 \mathrm{D}$ effective theory is determined by overlap integrals of zero-mode wavefunctions on each of the three $2 \mathrm{D}$ tori. The first one is given on the torus where the $6 \mathrm{D}$ fields live, and this part is represented by eq. (2.7). The magnetic fluxes on this torus will induce the hierarchical generation structure. On the other two tori, the zero-mode wavefunction of $10 \mathrm{D}$ field is still expressed by eq. (2.3), but the zeromodes of the other two bifundamental representations are not. The point-like localization of the bifundamental fields should be expressed by a delta function. Introducing the delta function to express the localizations as a point seems to be also a sensible proposal in a D-brane picture of superstring theories [2]. Thus, the integration on the torus is typically 


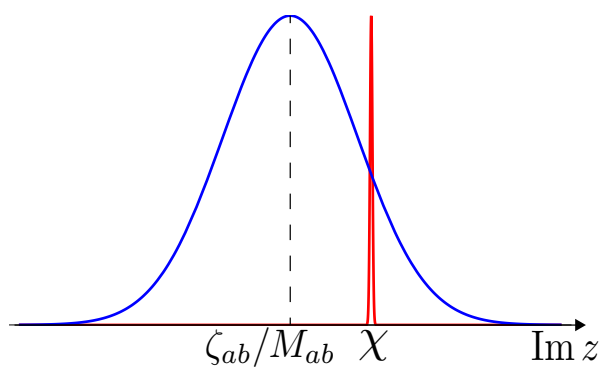

Figure 2. A schematic figure of the overlap integral (3.1) is shown. The blue Gaussian represents the wavefunction of $10 \mathrm{D}$ field and the red line expresses the point-like localization of $6 \mathrm{D}$ fields.

given by $[2,12]$

$$
g \int d z d \bar{z} \delta_{T^{2}}(z+\chi) \Theta^{I_{a b}, M_{a b}}\left(z+\zeta_{a b} / M_{a b}, \tau\right)=g \Theta^{I_{a b}, M_{a b}}\left(-\chi+\zeta_{a b} / M_{a b}, \tau\right),
$$

where the delta function $\delta_{T^{2}}(z+\chi)$ must satisfy the periodicity of torus, and $\chi$ indicates the localization point. The index $I_{a b}$ labels $\left|M_{a b}\right|$ flavors induced by magnetic fluxes on this torus, and this factor is universal for generations originating from the other tori.

This overlap (3.1) is schematically depicted in figure 2. This integration can take a tiny value when the peak position of $\Theta^{I_{a b}, M_{a b}}\left(z+\zeta_{a b} / M_{a b}, \tau\right)$ is far away from the point of magnetized torus indicated by $\chi$. That is, the distance $\left|\chi-\zeta_{a b} / M_{a b}\right|$ determines the magnitude of overlap. In the direction of $\operatorname{Im} z$, we can identify the coordinate as $\operatorname{Im} z \sim$ $\operatorname{Im} z+\operatorname{Im} \tau$. When the distance is given as $\left|\chi-\zeta_{a b} / M_{a b}\right| \sim \operatorname{Im} \tau / 2$, the suppression will be the strongest. Even when there is a deviation from $\left|\chi-\zeta_{a b} / M_{a b}\right|=\operatorname{Im} \tau / 2$, one can see from the figure that the strong suppression would also be obtained. Thus, we can realize tiny Yukawa coupling in a wide range of parameters.

We evaluate the suppression factor to verify consistency with neutrino masses, which roughly requires an $\mathcal{O}\left(10^{-10}\right)$ suppression [14]. For the purpose, we consider a mixture of a $6 \mathrm{D}$ theory compactified on a magnetized torus $\left(T^{2}\right)_{1}$ and a 10D SYM theory on magnetized $\left(T^{2}\right)_{1} \times\left(T^{2}\right)_{2} \times\left(T^{2}\right)_{3}$ in the following. When all the three generation structure of the SM is obtained on the first torus $\left(T_{1}^{2}\right)$, the other magnetized torus should not induce generations. This set-up implies $M_{a b}=1$ and $I_{a b}=1$ in the suppression factor (3.1) given on the second and the third tori. Such configurations can be indeed exist, and we will give a concrete model with such a configuration preserving $\mathcal{N}=1$ SUSY in the next section.

The suppression factor (3.1) on the $i$-th torus is a function of two parameters, $\tau_{i}$ and $X_{i} \equiv \zeta_{a b}^{(i)} / M_{a b}^{(i)}-\chi^{i}$. Note that, $\tau_{i}$ denotes the complex structure of the $i$-th torus, and $X_{i}$ represents a distance between the peak position of Gaussian-like wavefunction of a 10D field and a point at which other bifundamental fields are strongly localized by the delta function. In the analysis of this section, we neglect the prefactor of suppression which is composed by the gauge coupling $g$ and the normalization factor (2.6) originating from the function $\Theta^{I, M}$ as follows

$$
g \mathcal{N}_{1}=(2 \operatorname{Im} \tau)^{1 / 4} e^{\phi / 2} \sqrt{\frac{\alpha^{\prime}}{\mathcal{A}}}
$$




\begin{tabular}{|c|cc|}
\hline & case1 & case2 \\
\hline $\operatorname{Im} X_{2}$ & 15 & 7.5 \\
$\operatorname{Im} \tau_{2}$ & 30 & 15 \\
$f\left(X_{2}, \tau_{2}\right)$ & $1.2 \times 10^{-10}$ & $1.5 \times 10^{-5}$ \\
\hline $\operatorname{Im} X_{3}$ & 0 & 7.5 \\
$\operatorname{Im} \tau_{3}$ & 1.0 & 15 \\
$f\left(X_{3}, \tau_{3}\right)$ & 1.1 & $1.5 \times 10^{-5}$ \\
\hline$f\left(X_{2}, \tau_{2}\right) \times f\left(X_{3}, \tau_{3}\right)$ & $1.3 \times 10^{-10}$ & $2.3 \times 10^{-10}$ \\
\hline
\end{tabular}

Table 1. Function (3.2) is evaluated in two typical cases, which shows that the sufficient suppression to yield tiny neutrino masses is obtained with $\mathcal{O}(10)$ values of input parameters.

because this is typically of $\mathcal{O}(1)$ as long as the compactification scale is as high as $M_{\mathrm{GUT}}=$ $2.0 \times 10^{16}$. (In the next section, we also study the complete form of the Yukawa couplings given in a concrete model including these factors.) Thus, the suppression factor (3.1) can be rewritten as

$$
f\left(X_{i}, \tau_{i}\right) \equiv e^{\pi i X_{i} \operatorname{Im} X_{i} / \operatorname{Im} \tau_{i}} \sum_{\ell \in \mathbb{Z}} e^{\pi i \ell^{2} \tau_{i}} e^{2 \pi i \ell X_{i}}
$$

where we use the following property of wavefunction, $\Theta^{1,1}=\Theta^{0,1}$.

Our numerical analysis of the function (3.2) reveals that this factor can lead to tiny neutrino masses without strong fine tuning, which is shown in table 1. The suppression can be caused on the second and the third tori, and the $4 \mathrm{D}$ effective couplings contain their product, $f\left(X_{2}, \tau_{2}\right) \times f\left(X_{3}, \tau_{3}\right)$. In the case1, the second torus generates the tiny value of $f\left(X_{2}, \tau_{2}\right)$ and the other is of $\mathcal{O}(1)$. On the other side, both parts, $f\left(X_{2}, \tau_{2}\right)$ and $f\left(X_{3}, \tau_{3}\right)$, contribute evenly, where the value of input parameters become mild. In both cases, the values of input parameters are not so sensitive. With deviations of $( \pm 0.5)$ from the sample input values shown in the table, almost the same result would be obtained. In this analysis, we have assumed that the real parts of $X_{i}$ and $\tau_{i}$ are vanishing for simplicity.

\section{A concrete model}

We construct a specific model of the tiny neutrino Yukawa couplings respecting the minimal supersymmetric standard model (MSSM), on the basis of 6D and 10D SYM theories compactified on magnetized tori. A mixture of $6 \mathrm{D} \mathrm{U}(1)$ gauge theory compactified on $\left(T^{2}\right)_{1}$ and 10D U(7) SYM theory on $\left(T^{2}\right)_{1} \times\left(T^{2}\right)_{2} \times\left(T^{2}\right)_{3}$ is our starting point. If one would embed this system to string models, we have to care some string consistency. However, we cannot mention such stringy constraints now since we here propose a model of the visible sector and a hidden sector should be added into this system in order to describe our real world. 
We parametrize their magnetic fluxes as

$$
\begin{aligned}
M_{6 D} & =m \\
M_{10 D}^{(i)} & =\left(\begin{array}{ccc}
M_{C}^{(i)} \times \mathbf{1}_{3} & 0 & 0 \\
0 & M_{L}^{(i)} \times \mathbf{1}_{2} & 0 \\
0 & 0 & M_{R}^{(i)} \times \mathbf{1}_{2}
\end{array}\right),
\end{aligned}
$$

where $M_{6 D}$ represents the magnetic flux on the first torus in the U(1) gauge theory, and $M_{10 D}^{(i)}$ expresses those on the $i$-th torus in the U(7) SYM theory. In ref. [2, 13], conditions to preserve $\mathcal{N}=1$ SUSY out of the full $N=2,3$ and 4 in terms of the 4 D supercharges were studied, and it is given by

$$
m=0, \quad \sum_{i=1}^{3} \frac{M_{10 D}^{(i)}}{\mathcal{A}^{(i)}}=0,
$$

where $\mathcal{A}^{(i)}$ is the area of the $i$-th torus. This form of the magnetic fluxes generically break the gauge symmetry as $\mathrm{U}(7) \rightarrow \mathrm{U}(3)_{C} \times \mathrm{U}(2)_{L} \times \mathrm{U}(2)_{R}$. The last $\mathrm{U}(2)_{R}$ gauge should be further broken to $\mathrm{U}(1)_{R^{\prime}} \times \mathrm{U}(1)_{R^{\prime \prime}}$ by introducing Wilson lines of the form

$$
\zeta_{10 D}^{(i)}=\left(\begin{array}{cccc}
\zeta_{C}^{(i)} \times \mathbf{1}_{3} & 0 & 0 & 0 \\
0 & \zeta_{L}^{(i)} \times \mathbf{1}_{2} & 0 & 0 \\
0 & 0 & \zeta_{R^{\prime}}^{(i)} & 0 \\
0 & 0 & 0 & \zeta_{R^{\prime}}^{(i)}
\end{array}\right) .
$$

In this parametrization, $\mathrm{U}(7)$ adjoint representations are decomposed, which yield the MSSM fields except for the leptons. Bifundamental representations of $\mathrm{U}(7) \times \mathrm{U}(1)$ accommodates the charged lepton multiplets and the neutrino multiplets. Note that, the suppression factor is given to Yukawa couplings of the charged leptons as well as the neutrinos, but the distance $X_{i}$ for the each sector can be chosen independently. Thus, we can set them to give rise to just an $\mathcal{O}(1)$ factor for the charged leptons while realizing tiny neutrino Yukawa couplings.

We can find a configuration of magnetic fluxes, which leads to three generation structure for the quarks and leptons satisfying conditions (4.1) to preserve $\mathcal{N}=1$ SUSY, as

$$
\begin{aligned}
M_{6 D} & =0, \\
\left(M_{C}^{(1)}, M_{L}^{(1)}, M_{R}^{(1)}\right) & =\left(\begin{array}{ll}
0, & 3,-3
\end{array}\right), \\
\left(M_{C}^{(2)}, M_{L}^{(2)}, M_{R}^{(2)}\right) & =\left(\begin{array}{lll}
0,-1, & 0
\end{array}\right), \\
\left(M_{C}^{(3)}, M_{L}^{(3)}, M_{R}^{(3)}\right) & =\left(\begin{array}{lll}
0, & 0, & 1
\end{array}\right) .
\end{aligned}
$$

The SUSY condition is clearly satisfied with $\mathcal{A}^{1} / \mathcal{A}^{2}=\mathcal{A}^{1} / \mathcal{A}^{3}=3$. This configuration leads to three generations of $(3, \overline{2}, 1)$ and $(\overline{3}, 1,2)$ representation of $\mathrm{U}(3)_{C} \times \mathrm{U}(2)_{L} \times \mathrm{U}(2)_{R}$, which can be identified with the quark multiplets. Six generations of $(1,2, \overline{2})$ representation correspond to the Higgs multiplets. The existence of multiple Higgs fields is a generic 
feature of magnetized SYM models, and we identify a linear combination of them with the MSSM Higgs multiplets. In such multi Higgs models, we have to care about the flavor changing neutral currents, depending on its spectrum of the generations of Higgs fields. Although that is attractive phenomenological aspects of this model, we just assume here that the other five combinations are decoupled at a certain high-energy scale somehow, because it is not the purpose of this paper. The three generations of the charged lepton and neutrino multiplets are correctly given by the bifundamental representation charged under the original U(1) gauge group as we mentioned above. Thus, Yukawa couplings including the leptons have the global suppression factor (3.1).

Finally, we calculate the neutrino Yukawa coupling in this model. Its complete form is given by the overlap integrals on the three tori, and we find

$$
\begin{aligned}
Y_{I J K}= & g \prod_{i=1}^{3}\left(\frac{2 \operatorname{Im} \tau_{i}}{\left(A^{(i)}\right)^{2}}\right)^{1 / 4}\left(\frac{3 \times 3}{6}\right)^{1 / 4} \exp \left[\frac{-\pi}{\operatorname{Im} \tau_{1}} \frac{\left(\operatorname{Im} \zeta_{L R}\right)^{2}}{6}\right] f\left(X_{2}, \tau_{2}\right) f\left(X_{3}, \tau_{3}\right) \\
& \times \sum_{\ell \in \mathbb{Z}_{M_{a c}}} \vartheta\left[\begin{array}{c}
\frac{3(I-J)+9 \ell}{54} \\
0
\end{array}\right]\left(3 \zeta_{L R}, 54 \tau_{1}\right) \times \delta_{I+J+3 \ell, K},
\end{aligned}
$$

where we omit un-physical phases, and indices $I, J$ label the three generations of left- and right-handed neutrinos respectively, and the last index $K$ labels the six generations of up-type Higgs doublet. The difference of Wilson lines felt by the left- and right-handed neutrinos is denoted by $\zeta_{L R}$. In this expression, the global factor $f\left(X_{2}, \tau_{2}\right)$ and $f\left(X_{3}, \tau_{3}\right)$ appear. Note that, these factors also appear in the charged lepton sector, but the distance $X_{2}$ and $X_{3}$ can be chosen independently for the neutrino and charged lepton sectors. Thus we choose them as $\operatorname{Im} X_{i}=\operatorname{Im} \tau_{i} / 2$ for the neutrino sector to get the strong suppression, while we set as $\operatorname{Im} X_{i}=0$ for the charged leptons, which induces just a trivial factor $f\left(0, \tau_{i}\right) \sim 1$. As for the quark sector, the global factor is given by eq. (2.6) because of a vanishing flux, and there is no suppression.

In the assumption where one linear combination of the six Higgs doublets is identified with the MSSM Higgs field, the mass matrix of neutrinos is given by

$$
M_{I J}=\left\langle H_{u}^{(1)}\right\rangle Y_{I J 1}+\left\langle H_{u}^{(2)}\right\rangle Y_{I J 2}+\left\langle H_{u}^{(3)}\right\rangle Y_{I J 3}+\left\langle H_{u}^{(4)}\right\rangle Y_{I J 4}+\left\langle H_{u}^{(5)}\right\rangle Y_{I J 5}+\left\langle H_{u}^{(6)}\right\rangle Y_{I J 6}
$$

where $H_{u}^{(i)}$ is the $i$-th up-type Higgs doublet. These vacuum expectation values (VEVs) must be normalized as,

$$
\sum_{i=1}^{6}\left\langle H_{u}^{(i)}\right\rangle^{2}=v_{u}^{2},
$$

where $v_{u}$ represents the VEVs of the MSSM (up-type) Higgs field. We estimate the Yukawa coupling focusing on the difference of squared masses and we will not study the CP phases here. The relevant input parameters are as follows,

$$
g_{4 \mathrm{D}}, \operatorname{Im} \tau_{i}, \operatorname{Im} \zeta_{L R}, \operatorname{Im} X_{2}, \operatorname{Im} X_{3},\left\langle H_{u}^{(i)}\right\rangle
$$

where the four dimensional effective gauge coupling is defined by

$$
\frac{1}{g_{4 \mathrm{D}}^{2}} \equiv \frac{1}{g^{2}} \mathcal{A}^{(1)} \mathcal{A}^{(2)} \mathcal{A}^{(3)},
$$


and we take $\frac{4 \pi}{g_{4 \mathrm{D}}^{2}}=24$ in accordance with the unified value implied in the MSSM. Among these parameters, $\operatorname{Im} \tau_{1}, \operatorname{Im} \zeta_{L R}$ and $\left\langle H_{u}^{(i)}\right\rangle$ determine the texture of mass matrix, and the other give the suppression global factor.

We can demonstrate the tiny masses with the following input parameters,

$$
\begin{aligned}
& \operatorname{Im} \tau_{1}=2.0, \quad \operatorname{Im} \tau_{2}=\operatorname{Im} \tau_{3}=20, \\
& \operatorname{Im} \zeta_{L R}=0.5, \quad \operatorname{Im} X_{2}=\operatorname{Im} X_{3}=10, \\
& \left\langle H_{u}^{(1)}\right\rangle=\frac{0.8}{N_{u}} \times 170, \quad\left\langle H_{u}^{(2)}\right\rangle=\frac{2.8}{N_{u}} \times 170, \quad\left\langle H_{u}^{(3)}\right\rangle=\frac{1.3}{N_{u}} \times 170,
\end{aligned}
$$

where the normalization factor is given by $N_{u}=\sqrt{0.8^{2}+2.8^{2}+1.3^{2}}$ and the MSSM Higgs VEV is assumed as $v_{u}=170 \mathrm{GeV}$. We take for simplicity the other Higgs VEVs to be vanishing. With this ansatz, the mass eigenvalues and the differences are found as,

$$
\begin{aligned}
\left(M_{\nu_{1}}, M_{\nu_{2}}, M_{\nu_{3}}\right) & =\left(1.5 \times 10^{-5}, 8.1 \times 10^{-3}, 4.8 \times 10^{-2}\right) \mathrm{eV}, \\
\Delta m_{32}^{2} & =2.21 \times 10^{-3} \mathrm{eV}^{2} \\
\Delta m_{21}^{2} & =6.64 \times 10^{-5} \mathrm{eV}^{2} .
\end{aligned}
$$

These can be consistent with the observed values (normal mass hierarchy) even though the input parameters are chosen very roughly. (We have to slightly shift one of the parameters which affects on only the global factor in order to obtain exact consistency with experimental data [14]. ) We can also estimate the mass matrices of the quarks and the charged leptons in this model, and we will study the complete spectrum elsewhere.

\section{Conclusions and discussions}

We have studied strong suppression due to quasi-localization of wavefunctions and realized tiny neutrino Yukawa couplings in magnetized toroidal compactifications of SYM theories. In a system of two SYM theories defined in different dimensions, such as, 6D-10D SYM theories, bifundamental representations of the two gauge groups must be localized as a point in extra four directions, which should be represented by the well-defined delta function. As a result, their $4 \mathrm{D}$ effective couplings to $10 \mathrm{D}$ fields contain the factor induced by overlap integrals of the delta function and a Gaussian wavefunction of the 10D field in the four directions. When a point on which the delta function will not vanish is equal to the peak position of Gaussian, the integral induces an $\mathcal{O}(1)$ factor. However, for their nonvanishing distance, the value of the factor is reduced exponentially. Our numerical analysis shows that the $\mathcal{O}(10)$ of distance leads to an $\mathcal{O}\left(10^{-10}\right)$ of suppression, which is strong enough to yield tiny neutrino masses consistent with experimental and observational data.

A specific SYM system and its configuration have been discussed in section 4 . We consider a mixture of a $6 \mathrm{D} \mathrm{U}(1)$ gauge theory compactified on magnetized $\left(T^{2}\right)_{1}$ and a 10D U(7) SYM theory on $\left(T^{2}\right)_{1} \times\left(T^{2}\right)_{2} \times\left(T^{2}\right)_{3}$. The $\mathrm{U}(7)$ gauge group is broken in part by magnetic fluxes to $\mathrm{U}(3)_{C} \times \mathrm{U}(2)_{L} \times \mathrm{U}(2)_{R}$. The decomposed $\mathrm{U}(7)$ adjoint field accommodates the MSSM contents except for the lepton multiplets. The lepton multiplets are 
assigned into the bifundamental representation charged under the original (6D) U(1) gauge group. Thus, Yukawa couplings for the charged leptons and the neutrinos are determined by an overlap integral of three Gaussian wavefunctions on the first torus $\left(T^{2}\right)_{1}$, and by overlap integrals of the delta function and the Gaussian on $\left(T^{2}\right)_{2}$ and $\left(T^{2}\right)_{3}$. Since the distance $X^{i}$ between the peak of the delta function and the Gaussian can be controlled independently for the charged leptons and the neutrinos, neutrino Yukawa couplings can be strongly suppressed while just an $\mathcal{O}(1)$ factor is given rise to for the charged lepton sector. In this section, we also give sample values of neutrino masses. We have found that it is easily to obtain a realistic pattern of the masses.

This very strong suppression could be applied to other couplings to forbid dangerous processes, such as, the proton decay and the flavor violation. In magnetized toroidal compactifications, higher-order couplings can also be calculated analytically [15], and this strong suppression mechanism would also work in such higher-order couplings as well as Yukawa couplings. Thus, we expect that a wide variety of its applications are possible. We will construct models focusing on other phenomenologies and analyze them elsewhere.

Open Access. This article is distributed under the terms of the Creative Commons Attribution License (CC-BY 4.0), which permits any use, distribution and reproduction in any medium, provided the original author(s) and source are credited.

\section{References}

[1] C. Bachas, A way to break supersymmetry, hep-th/9503030 [INSPIRE].

[2] D. Cremades, L.E. Ibáñez and F. Marchesano, Computing Yukawa couplings from magnetized extra dimensions, JHEP 05 (2004) 079 [hep-th/0404229] [INSPIRE].

[3] N. Arkani-Hamed and M. Schmaltz, Hierarchies without symmetries from extra dimensions, Phys. Rev. D 61 (2000) 033005 [hep-ph/9903417] [INSPIRE].

[4] H. Abe, T. Kobayashi, H. Ohki, A. Oikawa and K. Sumita, Phenomenological aspects of $10 D$ SYM theory with magnetized extra dimensions, Nucl. Phys. B 870 (2013) 30 [arXiv: 1211.4317] [INSPIRE].

[5] H. Abe, J. Kawamura and K. Sumita, The Higgs boson mass and SUSY spectra in 10D SYM theory with magnetized extra dimensions, Nucl. Phys. B 888 (2014) 194 [arXiv:1405.3754] [INSPIRE].

[6] T. Yanagida, Horizontal symmetry and masses of neutrinos, in the proceedings of the Workshop on unified theory and baryon number in the universe, O. Sawada and A. Sugamoto eds., KEK, Tsukuba, Japan (1979).

[7] T. Yanagida, Horizontal symmetry and masses of neutrinos, Prog. Theor. Phys. 64 (1980) 1103 [INSPIRE].

[8] M. Gell-Mann, P. Ramond, R. Slansky, Complex spinors and unified theories, in Supergravity, D.Z. Freedman and P.van Nieuwenhuizen eds., North Holland, Amsterdam, The Netherlands (1979).

[9] L.E. Ibáñez and A.M. Uranga, Neutrino Majorana masses from string theory instanton effects, JHEP 03 (2007) 052 [hep-th/0609213] [INSPIRE]. 
[10] M. Cvetič, R. Richter and T. Weigand, Computation of D-brane instanton induced superpotential couplings: Majorana masses from string theory, Phys. Rev. D 76 (2007) 086002 [hep-th/0703028] [INSPIRE].

[11] L.E. Ibanez and A.M. Uranga, String theory and particle physics: an introduction to string phenomenology, Cambridge University Press, Cambridge U.K. (2012).

[12] H. Abe, T. Horie and K. Sumita, Superfield description of $(4+2 n)$-dimensional SYM theories and their mixtures on magnetized tori, Nucl. Phys. B 900 (2015) 331 [arXiv: 1507.02425] [INSPIRE].

[13] J. Troost, Constant field strengths on $T^{2 n}$, Nucl. Phys. B 568 (2000) 180 [hep-th/9909187] [INSPIRE].

[14] Particle Data Group collaboration, K.A. Olive et al., Review of particle physics, Chin. Phys. C 38 (2014) 090001 [INSPIRE].

[15] H. Abe, K.-S. Choi, T. Kobayashi and H. Ohki, Higher order couplings in magnetized brane models, JHEP 06 (2009) 080 [arXiv: 0903.3800] [INSPIRE]. 